\title{
From Usability Testing to Clinical Simulations: Bringing Context into the Design and Evaluation of Usable and Safe Health Information Technologies
}

\section{Contribution of the IMIA Human Factors Engineering for Healthcare Informatics Working Group}

\author{
A. Kushniruk' ${ }^{1,2}$, C. Nohr' ${ }^{2}$ S. Jensen², E. M. Borycki ${ }^{1}$ \\ I School of Health Information Science, University of Victoria, Victoria, Canada \\ ${ }^{2}$ Aalborg University, Department of Development and Planning, Aalborg, Denmark
}

\begin{abstract}
Summary
Objectives: The objective of this paper is to explore human factors approaches to understanding the use of health information technology (HIT) by extending usability engineering approaches to include analysis of the impact of clinical context through use of clinical simulations.

Methods: Methods discussed are considered on a continuum from traditional laboratory-based usability testing to clinical simulations. Clinical simulations can be conducted in a simulation laboratory and they can also be conducted in real-world settings. The clinical simulation approach attempts to bring the dimension of clinical context into stronger focus. This involves testing of systems with representative users doing representative tasks, in representative settings/environments.

Results: Application of methods where realistic clinical scenarios are used to drive the study of users interacting with systems under realistic conditions and settings can lead to identification of problems and issues with systems that may not be detected using traditional usability engineering methods. In conducting such studies, careful consideration is needed in creating ecologically valid test scenarios. The evidence obtained from such evaluation can be used to improve both the usability and safety of HIT. In addition, recent work has shown that clinical simulations, in particular those conducted in-situ, can lead to considerable benefits when compared to the costs of running such studies. Conclusion: In order to bring context of use into the testing of HIT, clinical simulation, involving observing representative users carrying out tasks in representative settings, holds considerable promise.
\end{abstract}

\section{Keywords}

Ussability, clinical simulation, patient safety, technology-induced error, health information systems

Yearb Med Inform 2013:78-85

\section{Introduction}

The usability of health information technology (HIT) has been increasingly recognized as being of critical importance in the design and deployment of systems that are both effective and acceptable to end users. Despite the considerable potential range of healthcare applications such as electronic health records, clinical decision support systems and consumer e-Health applications, there continues to be many reports of HIT that are unusable, that do not fit the workflow of users and that do not end up being adopted. In addition, in recent years, the relation between poor usability and unsafe systems has begun to be better understood [1,2], with growing evidence that highly unusable systems may not only be bothersome in terms of end user dissatisfaction, but that poor usability may underlie and lead to safety incidents and potential patient death [3-5]. Furthermore, recent studies have indicated that the extent of such problems may have been under-reported and may be more prevalent than previously thought [3-5]. All this is despite the increased consideration, discussion and publication of work and research aimed at improving the usability and safety of HIT, particularly over the past two decades [1, 3, 4, 6-9].

Although many vendors of HIT now operate commercial usability laboratories where healthcare software systems products are designed and tested, reports of serious usability and safety issues continue to be reported globally [3-5,10]. The question therefore arises as to why, despite this in- creased understanding and attention given to usability, reports of unusable and potentially unsafe systems are still appearing [3-5]? In this paper we will examine the need for considering the impact of context of system use on the ultimate usability and safety of HIT. Indeed, health informatics researchers and professionals have argued that of all work domains, healthcare is the most challenging given the variety, range and complexity of situations and settings where HIT is used and deployed [11]. Furthermore, a lack of understanding of the nature and range of end users of HIT has been highlighted as being a key issue in the failure of many systems to be adopted by health professionals and consumers [12]. Along these lines, understanding the context in which systems will be used must take into account not only tasks and settings but also the range, capabilities and cognitive capacities of an ever growing variety of potential end users [13-17].

In recent years, methodologies have begun to emerge where the design and evaluation of complex HIT has focused on an improved understanding of how such systems will work and be used in a range of complex and challenging settings. These settings typically vary considerably from those where healthcare software products are initially developed and tested (i.e. vendor usability laboratories and beta test sites at a few selected healthcare organizations) $[1,15,18]$. As will be discussed in this paper, this movement represents an extension of "traditional" laboratory usability engineering approaches to methods 
that incorporate evidence about system use under "near-live" or "live" conditions (in a range of settings and healthcare contexts) prior to widespread system deployment. It will also be shown how such methods are becoming increasingly used by organizations to obtain evidence about fit of commercial systems to their organizations in an effort to procure systems that more closely match organizational requirements, end user needs, and local healthcare practices [e.g. 19-22].

\section{From Usability Testing to Clinical Simulations and Rapid In-Situ System Testing: Bringing Context to the Fore}

Since the early 1990's a considerable number of published works have appeared describing the application of usability engineering methods to improving HIT. This has included description of the application of "classic" methods such as usability testing and usability inspection methods for improving the design and usability of a wide range of HIT [7, 8, 15]. Usability testing, as it has been defined in these works, typically refers to the observation of representative users of a system (or user interface) interacting with that system while carrying out representative tasks [15]. For example, in a range of studies of users of electronic health records (EHRs), this has involved observing and video recording health professionals (e.g. doctors and nurses) as they interact with EHRs in carrying out data entry, retrieval and decision making tasks $[1,13,14]$. The results of such study have been reported to have improved the design and adoption of many healthcare information systems [19, 20]. Complementary methods of usability inspection, including heuristic evaluation and cognitive walkthrough, have also been reported as being increasingly used in evaluating and testing a variety of health information systems [23, 24]. Unlike usability testing (which involves observing end users of a system), usability inspection methods have been noted for being particularly cost effective in that they are conducted by an analyst and do not involve testing of human users of a system [23, 24]. Indeed, in initial work in applying usability engineering to HIT, it has been assumed by many that methods involving observation of users were more labour intensive and costly than usability inspection. The assumption that conducting usability testing (typically involving video analysis and coding) is expensive has been challenged recently as described below when considering evidence from cost-benefit analyses of rapid usability testing methods in healthcare $[27,28]$.

\section{Low-cost Rapid Usability Testing: Adding Context by Bringing Testing to the Setting of Use}

In order to make usability testing more relevant by bringing testing of systems to contexts closer to real system use (than might be achieved in fixed usability laboratories) a range of approaches have appeared. These approaches typically involve free or very low cost screen recording software that can be installed on one or more computers in the actual environment where a system will be deployed [25]. This has typically involved having participants from the organization where the system will be deployed (e.g. health professionals) interact with the system under study in its naturalistic context and interfaced with other technologies it will be deployed with [e.g. 1, 25]. Scripts are typically created for "driving" the usability testing, whereby participants may be instructed to carry out specific tasks using the system under study, while their interactions are recorded (i.e. screens and physical interactions with the HIT, as well verbalizations) $[1,15,25]$. Participants might be instructed to "think-aloud" while carrying out tasks, as has become standard practice in many usability laboratories, but here the studies are conducted in the real environment of deployment, thereby decreasing costs associated with fixed laboratories while increasing ecological validity $[13,15,25,26]$. The interpretation and analysis of resultant data from such studies can vary in complexity but it has been found that even surface level analysis of resultant video can lead to identification of serious usability problems $[1,15]$.
Results from studies involving commercial systems already in deployment may lead to recommendations that feedback to: (a) the implementation team, who may be able to customize the system to mitigate identified usability problems whenever possible, (b) the team that trains new users, if problems found are best dealt with through training, (c) the vendor of the product, especially when problems found must be fixed as they are of an important or safety critical nature [27]. The economic benefits of conducting this type of analysis has recently been demonstrated by Baylis and colleagues [27] who found that a modest investment of under $\$ 5,000$ could lead to benefits (in terms of finding and fixing usability problems prior to widespread system release) in the order of five to ten times the cost of conducting the study [25,27]. Furthermore, when the potential consequences of usability errors that could lead to adverse patient events are taken into account, the benefits from such study increase considerably [27]. In another recent study examining the impact of this approach to usability testing in improving usability and refining the workflow of a tele-health decision support system in Canada, researchers found that usability analysis of users interacting with the system led to highly significant reductions in time per tele-triage calls [28]. Such studies, conducted in live settings (i.e. "in-situ" usability testing) have therefore been shown to be highly cost effective, much as usability inspection methods (which do not involve recruiting end users) have previously been shown to be cost-effective and therefore labelled as "discount" usability methods by Nielsen and others [25, 26].

The location of such testing can be in a range of settings. These locations can vary to the extent that the environment of the testing matches the environment in which the system being tested will actually be deployed (see Figure 1). For example, many facilities where HIT systems are tested could be considered "Laboratory" environments, including conventional usability testing laboratories at centralized conformance testing sites. Clinical simulations are located in the middle part of Figure 1, which we define in this paper as an extension of usability testing (which involves observing "representative users" doing "representative 
tasks"). Clinical simulations include a third dimension, namely "representative settings" $[1,17,19,20,28]$. Clinical simulations may be conducted in a laboratory setting (as will be described in the next section of this paper $[16,17])$ or in the actual real settings in which HIT will be deployed, which will be described in a subsequent section of this paper). From the far right side of Figure 1, we can see that recording of users interacting with a system under study can also take place in the "live" environment where the system has been deployed, which we have labelled as "naturalistic" studies in Figure 1. In some studies [ e.g. 29, 30] this may simply involve continuing to record user interactions from clinical simulations being conducted "in-situ" (i.e. in the real setting of system use), but doing so after the system has actually gone "live" and is being used for real patient care.

As illustrated in Figure 1, in moving from pure laboratory-based studies (depicted on the left hand side of the figure) to testing of systems that brings into consideration context to a greater degree, the possibilities range from conducting realistic testing using laboratory-based clinical simulations to clinical simulations conducted in real settings where systems will ultimately be deployed. Recent work in advancing clinical simulation methods in healthcare will be discussed in the remainder of this paper, beginning with an example of an advanced simulation laboratory in Denmark followed by discussion of work in conducting in-situ clinical simulations. In both types of testing the role and importance of context of system use is critical and is a focus of understanding the impact of system deployment.

\section{Addressing Clinical Context in a Simulation Laboratory: Experiences from the ITX-lab in Copenhagen}

In the Capital Region of Denmark clinical simulation has been used since 2007 to evaluate clinical information systems prior to implementation in hospitals in the region. In 2007 the IT Experimentarium (ITX) was established with the purpose of strengthening the quality and optimizing the use of clinical information systems by using clinical simulation. The clinical simulations take place at the Danish Institute for Medical Simulation (DIMS) at one of the major university hospitals in Copenhagen (i.e. the Herlev Hospital). The top floor of the hospital is equipped with 13 simulation rooms consisting of the following: ordinary bed rooms, intensive care units, operating room, and a medication room (most of them with a control room separated with a one way mirror). Each simulation room is equipped with computer-controlled mannequins representing patients of all ages from babies to adults, remote controlled ceiling mounted video cameras, loud speakers, microphones and intercoms linked to the control room. The facilities are mainly used for clinical training, but the laboratory facilities (in conjunction with the use of clinical simulations) have also been employed in the development and evaluation of clinical information systems. Figure 2 shows the simulation room as it is seen from the control room through the one-way-mirror. Here, a simulation is being undertaken where there are two patients in a simulated hospital room. Figure 3 is a more complex example of a simulation. In Figure

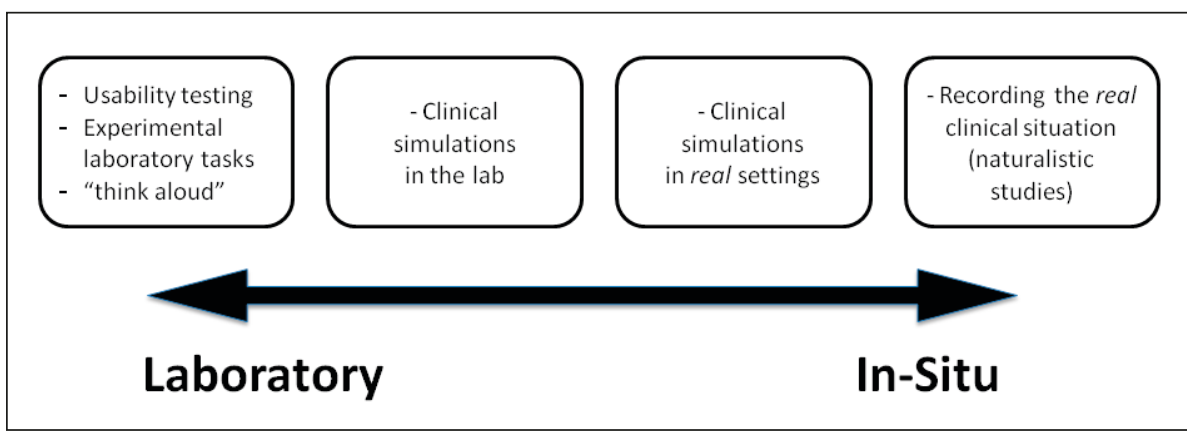

Fig. 1 The context of system testing - a continuum from laboratory to naturalistic settings.
3 an operation is being simulated. Included in the simulation is the equipment typical of an operation as well as the HIT. During the simulation the people in the control room are able to communicate with actors playing patients. The computer screens used by the physicians and nurses are mirrored in the control room.

The clinical simulations have appeared to be cost-effective, and since 2011 it has become mandatory to conduct clinical simulation tests before new systems that affect clinical work practices are implemented. During the last 5 years more than 20 simulation studies have been performed in the ITX-lab to improve HIS development activities and assist in the evaluation of clinical information systems [31, 32]. The simulation studies have been used to design computerized clinical decision support and standardized nursing documentation as well as to evaluate the impact of innovative technology.

In the ITX-lab clinical simulation studies are performed in representative realistic environments with real end-users (see Figures 2 and 3). Before the actual simulations take place, the scenarios are created, and the design of the evaluation study is developed. Depending on the maturity of the information system being tested, test data and technical environments must be prepared and implemented. The resources spent on preparing simulation studies can be quite exhaustive and depend on the requested degree of fidelity (i.e. the degree of fidelity must therefore be carefully chosen). The actual simulation with end-users, however, is not so time consuming. By preparing the clinical and technical set-up carefully, the time spent by physicians and nurses is often not more than a couple of hours (depending on the scenario and evaluation set-up).

The simulations are performed in three phases. When the clinicians arrive, they are introduced to the information system and to the simulation. They normally get some hands-on experience with the system before the simulation starts. Each simulation is observed by health informatics experts and sometimes by key stakeholders, such as colleagues from hospitals, clinical managers, quality managers and vendors. Depending on the purpose of the clinical simulation, the clinicians are sometimes also able to observe their colleagues, when not participating in the simulation themselves. After 


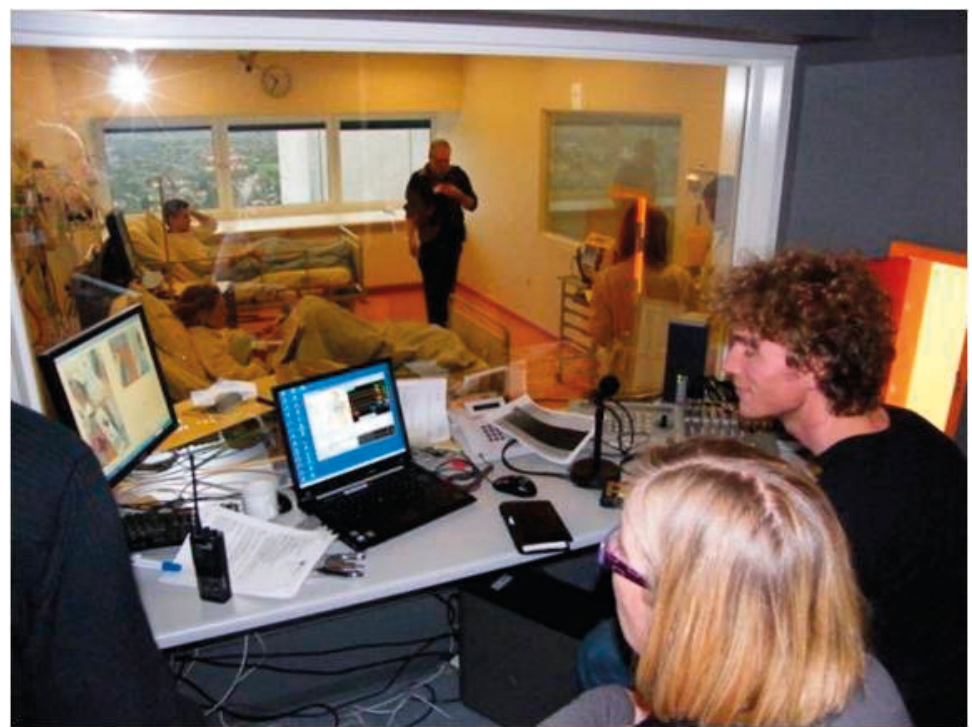

Fig. 2 Simulation room seen from the control room.

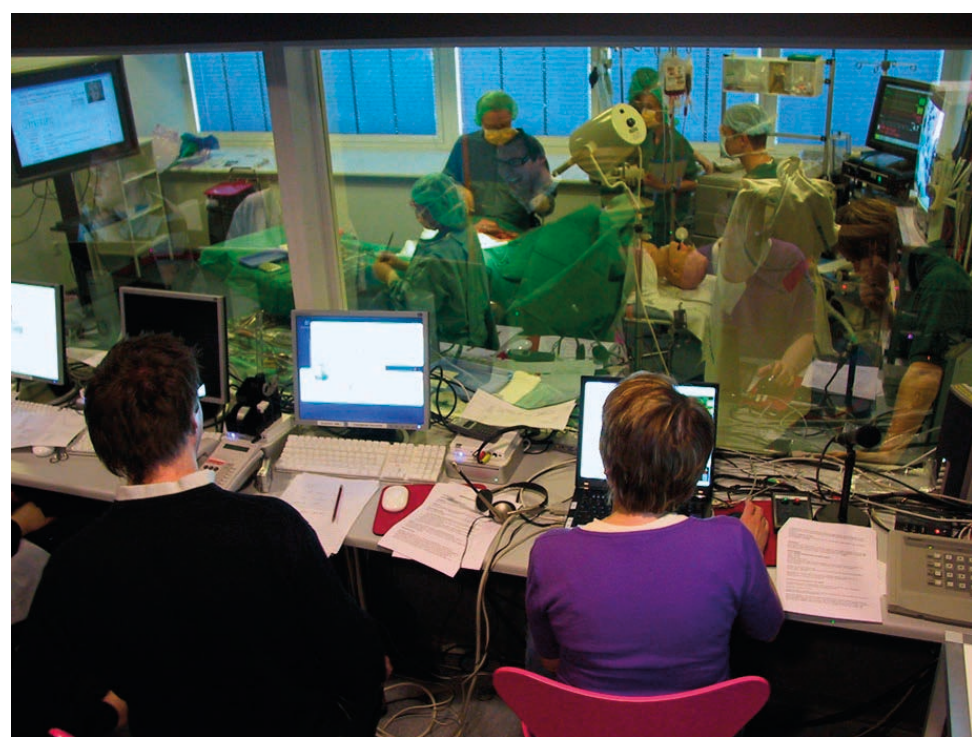

Fig. 3 Complex simulation of an operating room environment.

experienced [34-36]. In the design phase, clinical simulations have been used to obtain consensus among differing stakeholders; e.g. end-users and the quality unit. Clinical simulations make it possible for different stakeholders to observe new technology in use. The interviews and discussions that follow clinical simulations provide an opportunity for obtaining and understanding work practices and user needs. Clinical simulations may therefore help to reveal divergences of opinion among differing stakeholders and may make it possible to discuss and gain a common understanding of other stakeholders' views.

Clinical simulations have also been used as part of a participatory design approach making stakeholders actively involved in the design activities and thereby allowing stakeholders to influence HIT design solutions. In this case the clinical simulations were preceded by several design workshops with all stakeholders, where prototypes were built. The clinical simulations were performed by clinicians, who had not taken part in the workshops and therefore it was possible for evaluators to assess the effect of the prototype upon clinical work practices. Before an actual implementation takes place, clinical simulation makes it possible to assess health professional training and information needs. Such knowledge concerning work practices and patient safety issues may be gained, and used as important inputs before or during a pilot implementation of a HIS.

the simulations are completed, an evaluation is performed. Participants are asked to complete questionnaires and participate in a de-briefing interview. Observations made by the observers during the simulations are used as background for the interviews. The interview and notes from the observers are analyzed by use of Instant Data Analysis (IDA) [33]. IDA is a cost-saving analysis technique which allows usability evaluations to be conducted, analyzed and documented in less than a day. In a case study it was discovered that IDA reduced the time required to do a video data analysis by $90 \%$. IDA also identified $85 \%$ of the critical usability problems in the evaluated system.
In the Capital Region of Denmark clinical simulations are used in all phases of the software development life cycle of clinical information systems, starting with analysis of user requirements through to the development, evaluation, and implementation phase of a HIT. Clinical simulation has been used to analyze user requirements with use of different degrees of clinical simulation fidelity; both in connection with well described scenarios and mature prototypes with realistic test data and in a more experimental way with use of a "wizard-of-oz" approach, where clinicians themselves describe the scenarios from a typical patient case they have recently
A wide range of results have emerged from the simulation work conducted in the ITX-lab. In one paper by Jensen and colleagues [35] new potential users and new potential ways of using HIT were discovered. The study also revealed unintended benefits regarding new technology as a powerful learning tool and revealed unintended organizational issues concerning terminology and staff responsibility. The study also demonstrated clinical simulations could provide input into HIT design and configuration. In another study [31] using a participatory design approach clinical simulation contributed to the inclusion of stakeholders from all levels of the organization and offered a visualization of future work processes in relation to the new technology. Clinical simulations assisted the participants to gain a shared mental model which helped to reach some kind of consensus in the design 
discussions. In a large European project concerning contextualized computerized decision support [36] clinical simulation led to important insights into the potential harmful effects of deployment of information technologies. In this study the effect of a prototype was assessed in the simulation-lab as the prototype was too immature to be implemented at a hospital. Clinical simulation was used in different phases of the project and also led to creation of design principles encapsulating central themes of different kinds of clinical decision support [34].

In addition, the ITX-lab will be used this summer as part of a major procurement process in the Capital Region of Denmark and Region Zealand. This work will include comparison of vendor EHR products and will involve evaluation of three tenders. During the clinical simulation 12 clinical key scenarios such as ward rounds, administration of drugs, admission to and discharge from the hospital will be simulated in realistic clinical settings. The assessment of the EHR vendor products will involve 18 healthcare actors who are physicians and nurses from different specialties. The assessment is expected to run in three parallel tracks over a period of 10 days. The results of the simulation testing of the three products will be used in the decision making process for system selection.

\section{Addressing Clinical Context in In-Situ Testing}

In addition to testing of systems within simulation laboratories as described in the previous section, there is also a growing need to evaluate systems within the live contexts in which they will ultimately be deployed, with much of the interest in this direction coming from the desire to ensure system safety [9]. A variety of studies have been conducted in real settings such as hospital rooms, operating rooms and clinical settings. In many cases this has involved setting up clinical simulations (including full computer screen recording and video recording of participants) within hospitals, clinics and even home settings [16, $19,29]$. For example, in one study of the use of a new medication administration system in a hospital in Japan, a typical hospital room was secured for the study. The study was undertaken over several days. Technologies that were to be interfaced with the new system, such as a bar code scanner, were also included in the study to increase its realism. A set of scenarios were created to drive the simulations. Participants in the study (i.e. nurses and physicians) were asked to carry out the tasks defined by the scenarios and were video recorded as they interacted with a "dummy" patient (i.e. a mannequin) and the computer system to carry out medication administration tasks [9]. From this work, it was found that key aspects of the system needed to be customized prior to releasing the system widely in the hospital it was to be deployed in. For example, from the simulation testing, the system was found to be generally safe except when a patient emergency occurred that required the health professional to "break out of" the rigid sequence of steps imposed by the system when time did not allow for the workflow imposed by the system. Based on these results, an emergency override capability was added to the system, to be used only during emergency situations where a health professional would not have enough time to complete the prescribed work sequence of the computer system. It should be noted that this type of evaluation tested the system under the conditions typical of the institution where it was to be deployed, including working with all interfacing technologies. In addition, as it was conducted off hours in a hospital room it did not require use of an expensive laboratory setting, but rather included the context of the real setting at a low cost. Also, the realism of the setting allowed for system evaluation that included analysis of how the system interacted with situational factors (e.g. room size, frequency of emergency situations, bar code scanning technology etc.).

\section{Increasing Ecological Validity: from Clinical Simulations to Naturalistic System Testing}

An important aspect of clinical simulation is ecological validity. "Ecological validity refers to an acknowledgment of the fact that human action is situated and highly contingent on contextual factors/variables." Therefore, "to obtain 'valid' results, humans should be studied in the richness of their natural environment" [37]. When an environment is ecologically valid, the research setting matches the real-world setting. This "real-world" match ensures that the problem under study can be fully described and understood [38]. Clinical simulations provide an opportunity for this. Therefore, ecological validity is an important part of clinical simulations. For a clinical simulation to be ecologically valid, attention needs to be paid to setting (i.e. environment), task, users, and scenario representativeness (see Figure 4).

Such realism is critical as it ensures the results of the study are generalizable to the real-world. The more realistic the clinical simulation, the more generalizable and use-

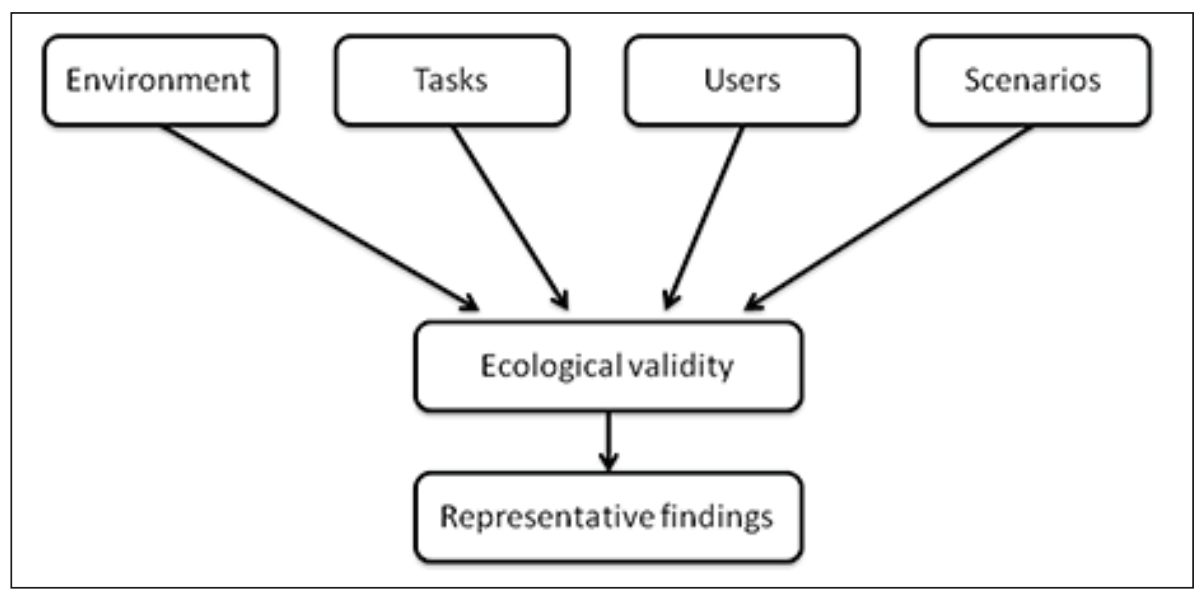

Fig. 4 Aspects of a clinical simulation that ensure it is ecologically valid. 
ful its results will be to real-world, systems designers, developers and implementers $[13,17,38]$. For example, in designing a clinical simulation there is a need to address the setting or environment, where the study will take place [39]. Whether one is conducting a clinical simulation in a traditional laboratory setting (i.e. a room set aside for clinical simulations) or in-situ (i.e. in a hospital room or clinic room that is not being used at the moment), an emphasis should be placed on ecological validity. In a laboratory setting this involves identifying the medical equipment (e.g. intravenous pumps, hospital beds, bedside tables, blood pressure monitoring cuffs etc.) that are the same or similar to the ones that will be used in the clinical setting where the software will be implemented. In addition to this, the hardware that is used in that setting will also need to be used in the clinical simulation. This may include desktop or laptop computers, tablet devices or mobile/ Smart phones that are currently being used or will be used in that setting (should there be a need for testing). In addition to this, existing software (currently being used) and the new or newly customized software that will be introduced to the setting will need to be included in the clinical simulation. Here, there is an emphasis on replicating the existing setting as well as knowing what hardware and software will be introduced to the setting to determine its effects on health professional work. Following this, there is a need to identify what tasks will be undertaken using the newly introduced hardware and/or software. Tasks should include those that users will be expected to perform using the new technology. A range of tasks should be selected: from the routine to the complex, from the typical to the atypical and from the non-urgent to urgent (see Table 1 for examples).

To illustrate, we use the example of a physician order entry system that will be tested using a clinical simulation approach. Here, users (i.e. physicians) are asked to undertake a range of physician order entry tasks using the new system. In addition to this, scenarios need to be developed to fully simulate the conditions under which the software will be used. Scenarios should be realistic and representative of the types of situations that would be encountered in the clinical setting.

Table 1 Examples of tasks using computerized physician order entry.

\begin{tabular}{|c|c|}
\hline Task Type & Example \\
\hline Routine to complex: & $\begin{array}{l}\text { Routine: Entering a single oral medication order. } \\
\text { Complex: Entering } 5 \text { oral medication orders, an order for an intravenous } \\
\text { medication and an order for a subcutaneous injection. }\end{array}$ \\
\hline Typical to atypical: & $\begin{array}{l}\text { Typical: Entering an order for a blood pressure medication. } \\
\text { Atypical: Entering orders for a chemotherapy regime for a rare cancer. }\end{array}$ \\
\hline Urgent to non-urgent: & $\begin{array}{l}\text { Non-urgent: Entering an order for a Tylenol to be taken as needed. } \\
\text { Urgent: Entering an order for a stat medication. }\end{array}$ \\
\hline
\end{tabular}

Therefore, much like tasks, scenarios must also range from the routine to the complex, from the typical to the atypical and from the urgent to the non-urgent (see Table 1 for examples). A range of scenarios need to be tested. Scenarios can be developed with the planned users of the new technologies and technology-implementers. This work can be done in the context of focus groups. Lastly, there is a need to ask representative users to participate in clinical simulations. Users should represent all health professionals who will be using the technology and should include novices, intermediates and experts (in an area of disciplinary practice - e.g. physician, nurse; in the domain of practice - e.g. medicine, surgery, neurology; in working with technology that will be used - e.g. new mobile device, new physician order entry system) [13]. For example, novice through to expert physicians should be asked to participate in the clinical simulations as well as novice through to expert hardware and software users to fully understand the implications of introducing new software and/or hardware in a clinical setting. In summary, ecological validity is an important aspect of designing clinical simulations. Attention to the ecological validity of a simulation ensures that the results can provide significant insights when a new technology (i.e. hardware/software) is used.

Recent work reported by Li and colleagues [29] illustrates some of the types of results that can be collected from using realistic clinical cases and simulations. In their study, the objective was to optimize clinical decision support embedded within a commercial EHR system, taking context of use into account. Their study involved three phases: (1) a standard usability test of the user interface driven by a script that led users through its functions for two clinical cases, (2) a clinical simulation where participants interacted with a digital patient (i.e. recording of patients with different respiratory problems), (3) naturalistic recording of physicians interacting with the system just prior to widespread system release. It was found that the three layers of testing led to different results which were all used to optimize the decision support tool for use within the EHR. For example from the initial usability testing it was found that physicians did not recognize the terminology used in the user interface (i.e. labels for buttons) and as a result did not access the decision support. Based on that and related findings the interface was optimized and the second phase of testing began - i.e. the clinical simulation. During the clinical simulations it became clear that the decision support was not being triggered (i.e. invoked) at the points in clinician workflow that the designers have expected. As a result of this finding, the integration of the decision support tool in the EHR was modified to lead to more appropriate invocation. Finally, during the final phase of naturalistic testing (in the "near live" environment) it was found that certain features of the user interface and its integration with clinician workflow needed further optimization. After undergoing these layers of testing the decision support tool was widely released, with a high level of uptake by end users and a high rate of acceptance of recommendations made by the system [29]. 


\section{Comparing Approaches: Trade- offs in Selecting Methods}

A wide range of approaches to evaluation of HIT have emerged based on methods from the human factors literature [40]. As described in this paper, a number of the approaches, including usability testing methods, have been used and adapted for evaluation of HIT [15]. In addition, we have seen the emergence of new types of testing, for example, clinical simulations, which borrow from the underlying human factors literature but also specialize the methods and techniques further for application in the complex domain of healthcare. Given the range of approaches possible, consideration of trade-offs in selecting methods for evaluating HIT is an important issue [41]. In this paper we have described conducting usability testing and clinical simulations in both laboratory and naturalistic settings. Obviously the availability of facilities such as fixed usability or simulation laboratories is one major consideration, and when such facilities are available they can form an important hub or centre for carrying out usability engineering methods with HIT $[34,35,36]$. Such work can entail testing systems throughout the system development life cycle, from prototype to completed system. In addition, a new area of testing HIT emerged during the procurement and selection process of systems such as EHRs. However, it has also been shown that low-cost portable approaches can also be employed to carry out studies that range from laboratory-based through to naturalistic evaluations [15, 25, 29]. Furthermore, when systems need to be tested in the actual environment they will be used (to ensure both usability and safety) low-cost portable methods have the advantage of being able to be conducted in the real (or close to real) setting of system use (along with all interfacing technologies). In the future we hope to see more integration of varied testing methods, including conformance testing of HIT at central laboratories in conjunction with localized testing in real settings and contexts of use. It is argued that only with such a combined approach will the usability and safety of HIT improved.

\section{Towards Increased Consider- ationof Context: The Context Sensitive Health Informatics Pre-Medinfo 2013 Conference}

As outlined above, human factors / ergonomics adopts a system-based approach to understanding and explaining the interactions between humans and other elements of a health care system. Humans approach tasks and systems with perspective, experience, knowledge, skills and preferences. The Human Factors approach is distinctly design driven and aims to optimize performance, safety and users' sense of well-being associated with their use of a system through the application of user-centred systems design and evaluation. On a health care system level, the socio-technical perspective maintains that the health information system integrates the human, social, organizational and technological dimensions and in so doing contributes to an essential body of knowledge of existing healthcare systems and contributes to their continuous evolution.

The design, implementation, and evaluation of safe, effective, efficient and easy to adopt HIT, therefore requires proper consideration of human and organizational factors. Health care organizations, health policy makers and regulatory bodies globally are starting to acknowledge this essential role of human and organisational factors and progressively incorporate them into regulations and safety initiatives. The two perspectives (Socio-technical and HF/E) are highly complementary to each other in terms of their methods, concepts, models and recommendations. Both contribute to a common body of knowledge and evidence allowing a better understanding of the reasons for success or failure in the Health Informatics and HIT domains, and more importantly opening the way for more efficient and safer practices in design, implementation, usage and evaluation of HIT.

The pre-Medinfo conference "Context Sensitive Health Informatics" merges and continues two conference series: ITHC -Information Technology in Health Care: Socio-Technical approaches previously held in 2001 (Rotterdam, Netherlands), 2004
(Portland, USA), 2007 (Sidney, Australia) and 2010 (Aalborg, Denmark) and HFE-HI: Human Factors / Ergonomics for Health Informatics previously held in 2006 (Lille, France), 2007 (Aarhus, Denmark), 2008 (Amsterdam, Netherlands), 2009 (Sonoma, USA) and 2011 (Trondheim, Norway). The conference will be held on August 17-18, 2013 in Herlev Hospital in Copenhagen. For more information see the conference web-site: www.cshi2013.org

\section{Conclusions}

The usability of HIT has been increasingly recognized as being of critical importance in the design and deployment of systems that are both effective and acceptable to end users. In this paper we have discussed several approaches to collecting evidence about the impact of HIT deployments. It was noted that there appears to be a trend in the human factors in healthcare literature towards increasing the ecological validity of system testing by bringing consideration of context into greater focus. Different approaches have been described in terms of a continuum that runs from laboratory-based usability testing to clinical simulations and testing of systems in their naturalistic environments. While conventional laboratory-based usability testing is an important component of ensuring that systems are usable and safe, it has been argued that they may be insufficient to ensure usability and safety once systems are released in real healthcare settings. Therefore, complementary methods are needed, along with the need for development and dissemination of new low-cost approaches that can be applied widely within healthcare organizations and settings to provide healthcare organizations, systems developers and customizers with evidence about how HIT will impact healthcare processes and patient safety in differing contexts of use.

\section{References}

1. Kushniruk AW, Triola MM, Borycki EM, Stein B, Kannry JL. The relationship between usability problems and prescription errors when using a handheld application. Int J Med Inform 2005;74(7- 
8):519-26.

2. Koppel R, Metlay JP, Cohen A, Abaluck B, Localio $\mathrm{AR}$, Kimmel SE, et al. Role of CPOE in facilitating medication errors. J Am Med Inform Assoc 2005; 293(10):1197-203.

3. Magrabi F, Ong M-S, Runciman W, Coiera E. An analysis of computer-related patient safety incidents to inform the development of a classification. J Am Med Inform Assoc 2010;17:663-70.

4. Magrabi F, Ong M., Runciman W, Coiera E. Using FDA reports to inform classification of HIT safety problems. J Am Med Inform Assoc 2012;19:45-53.

5. Samaranayake NR, Cheug STD, Chui WCM, Cheung BMY. Technology-related medication errors in a tertiary hospital: A 5-year analysis of reported medication incidents. Int J Med Inform 2012;81:828-33.

6. Carvalho CJ, Borycki EM, Kushniruk AK. Ensuring the safety of health information systems. Healthc Q2009; 12:49-54.

7. Rasmussen R, Kushniruk, A. The long and twisting path: An efficiency evaluation of an electronic whiteboard system. Stud Health Technol Inform 2013;183:174-8.

8. Lilholt LH, Pedersen SS, Madsen I, Nielsen PH, Boye N, Andersen SK, Nohr C. Development of methods for usability evaluations of EHR systems. Stud Health Technol Inform 2006;124:341-6.

9. Borycki EM, Kushniruk, AW, Kuwata S, Kannry J. Engineering the electronic health record for safety: A multi-video-based approach to diagnosing and preventing technology-induced error arising from usability problems. Stud Health Technol Inform 2011;166;197-205

10. Beuscart-Zéphir MC, Pelayo S, Anceaux F, Meaux J, Degroisse M, Degoulet P. Impact of CPOE on doctor-nurse cooperation for the medication ordering and administration process. Int J Med Inform 2005;74(7):629-41.

11. Carvalho CJ, Borycki EM, Kushniruk AK. Ensuring the safety of health information systems. Healthc Q 2009;12:49-54.

12. Kushniruk A, Turner P. A framework for user involvement and context in the design and development of safe e-Health systems. Stud Health Technol Inform 2012;180:353-7.

13. Borycki EM, Lemieux-Charles L, Nagle L, Eysenbach G. Evaluating the impact of hybrid electronic-paper environments upon novice nurse information seeking. Methods Inf Med 2009;48(2):137-43.

14. Pearce C, Shachak A, Kushniruk A, de Lusignan S. Usability: A critical dimension for assessing the quality of clinical systems. Inform Prim Care 2009:17(4):195-8.

15. Kushniruk AW, Patel VL. Cognitive and usability engineering methods for the evaluation of clinical information systems. J Biomed Inform 2004:37(1):56-76.
16. Ammenwerth E, Hackl WO, Bintzer K, Christoffersen TEH, Jensen S, Lawton K, et al. Simulation studies for the evaluation of health information technologies: experiences and results. HIM J 2012;41(2):14-21.

17. Borycki E., Kushniruk A, Anderson J, Anderson M. Designing and integrating clinical and computer-based simulations in health informatics: From real-world to virtual reality. In: Cakaj S, editor. Modeling Simulation and Optimization: Focus on Applications.Vukovar, Croatia: In-Tech; 2010. p. 31-52.

18. Borycki EM, Keay L. Methods to assess the safety of health information systems. Healthc Q 2010:13,49-54.

19. Kushniruk AW, Borycki EM, Kuwata S, Watanabe $\mathrm{H}$. Using a low-cost simulation for assessing the impact of a medication administration system on workflow. Stud Health Technol Inform 2008:136:567-72.

20. Zheng K, Padman R, Johnson MP, Diamond HS. Understanding technology adoption in clincal care: Clinician adoption behaviour of a point-of-care reminder system. IntJ Med Inform 2005;74(7):535-43.

21. Kushniruk AW, Beuscart-Zephir MC, Grzes A Borycki E, Watbled L, Kannry J. Increasing the safety of healthcare information systems through improved procurement: Toward a framework for selection of safe healthcare systems. Healthc Q 2010;13:53-8.

22. Marcilly R, Bernonville S, Riccioli C, Beuscart-Zephir MC. Patient safety-oriented usability testing: A pilot study. Stud Health Technol Inform 2012;180:368-72.

23. Nielsen J, Mack R. Usability inspection methods. New York: John Wiley; 1994.

24. Sharp H, Preece J. Interaction design ( $3^{\text {rd }}$ ed.). New York: Wiley;2011.

25. Kushniruk AW, Borycki EM. Low-cost usability engineering. Healthc Q 2006; 9(4):98-100, 102.

26. Kushniruk, AW, Borycki, EM, Kannry, J. Commercial versus in-situ usability testing of healthcare information systems: Towards "public" usability tesing in healthcare organizations. Stud Health Technol Inform 2013;183:157-61.

27. Baylis T, Kushniruk AW, Borycki EM. Low-cost usability for health information systems: Is it worth the effort? Stud Health Technol Inform 2012;180:363-7.

28. Hall SA, Kushniruk AW, Borycki EM. Usability analysis of the tele-nursing call management software at HealthLink BC. Stud Health Technol Inform 2011:164:208-12.

29. Li AC, Kannry JL, Kushniruk A, Chrimes D, McGinn TG, Edonyabo D, Mann DM. Integrating usability testing and think-aloud protocol analysis with "near live" clincal simulations in evaluting clinical decision support. Int J Med Inform 2012;81(11):761-21
30. Kushniruk AW, Borycki EM, Kuwata S, Kannry J. Emerging approaches to usability evaluation of health information systems: Towards in-situ analysis of complex healthcare systems and envioronments. Stud Health Technol Inform 2011:169:915-9.

31. Rasmussen SL, Lyng KM, Jensen S. Achieving IT-supported standardized mursing documentation through participatory design. Stud Health Technol Inform 2012;180:1055-9.

32. Ammenwerth E, Hackl WO, Jensen S, Lawton K, Riedmann D, Jung M. Impact evaluation of innovative technology: estimating the impact of the PSIP solutions. Stud Health Technol Inform 2011;166:227-33.

33. Kjeldskov J, Skov MB, Stage J. Instant data analysis: conducting usability evaluations in a day. NordiCHI '04 Proceedings of the third Nordic conference on Human-computer interaction, ACM; 2004.

34. Jensen S, Kanstrup AM, Nohr C. User driven development and high fidelity testing. Proceedings Eleventh Danish HCI Research Symposium; 2011.

35. Jensen S, Vingtoft S, Nøhr C. Benefits of a clinical planning and coordination module: A simulation study. Stud Health Technol Inform 2013;183:220-4.

36. Lawton K, Bintzer K, Skjoet P, Jensen S. Lessons learned from conducting a high fidelity simulation test in health IT. Stud Health Technol Inform 2011; 166:217-26.

37. Interaction design. Ecological validity. [Internet]. Accessed from: http://www.interaction-design.org/ encyclopedia/ecological_validity.html

38. Elmes DG, Kantowitz BH, Roediger HL. Research methods in psychology. New York: Wadsworth Publishing Company; 2011.

39. Sanderson P. Designing and evaluating healthcare ICT innovation: A cognitive engineering view. Proceedings of the Third International Conference on Information Technology in Healthcare-Sociotechnical Systems. Sydney, 28-30 August, 2007.

40. Carayon P. Human factors and ergonomics in health care and patient safety (Second edition). New York: CRC Press; 2012.

41. Jaspers M. A comparison of usability methods for testing interactive health technologies: Methodological aspects and empirical evidence. Int J Med Inform 2009;78:340-53.

Correspondence to:

Dr. Andre Kushniruk

Health Information Science

University of Victoria

Human \& Social Development Building A202

3800 Finnerty Road (Ring Road)

Victoria, BC V8P 502

Canada

E-mail: andrek@uvic.co 